\title{
Transformaciones en la estructura socio-ocupacional de la provincia de Nuble: mirada desde la perspectiva funcionalista
}

Transformation in the structure socio-occupational of the Nuble province: outlook from the functionalist perspective

\author{
DR. HÉCTOR CÁRCAMO VÁSQUEZ \\ Doctor en Antropología Social y Cultural \\ Universidad del Bío-Bío, Chillán, Chile \\ hcarcamo@ubiobio.cl
}

\section{DR. PABLO MÉNDEZ BUSTOS}

Doctor en Psicología

Universidad Católica del Maule, Talca, Chile

pmendez@ucm.cl

\section{RESUMEN}

El artículo que se presenta tiene por finalidad determinar las transformaciones ocurridas en la estructura socio-ocupacional de la provincia de Ñuble en el período inter-censal 19922002. Para tal efecto, se trabajó desde la noción funcionalista, considerando el comportamiento de la población económicamente activa al interior de la estructura ocupacional. El diseño corresponde a un estudio cuantitativo de tipo longitudinal descriptivo, con base en la información secundaria. El resultado de mayor trascendencia indica que la provincia de Ñuble ha experimentado reformas en la categoría ocupacional en el período inter-censal 1992-2002, reconociéndose un importante cambio en la rama de actividad económica en la que se concentra la población económicamente activa.

Palabras clave: estructura socio-ocupacional, categoría ocupacional, población económicamente activa, funcionalismo. 


\section{ABSTRACT}

The aim of this article is to determine the changes happened in the socio-occupational structure of Nuble province in the period between 1992 and 2002 censuses. In order to achieve this purpose the functionalist notion was used considering the behavior of the population economically active in the interior of the occupational structure. The design corresponds to a quantitative study of descriptive longitudinal type based on secondary information. The most relevant result shows that the Ñuble province has undergone a change in the occupational category in the 1992-2002 inter census period pointing out an important transformation in the economically active population.

Key words: socio-occupational structure, occupational category, economically active population, functionalism.

\section{INTRODUCCIÓN}

Indistintamente del nivel de desarrollo alcanzado por las sociedades, siempre han existido determinados estilos de estratificación social, pero es precisamente en las sociedades industriales donde surge la inquietud de incorporarla como objeto de estudio, considerando como unidad de clasificación más general el concepto de clases.

El estudio de la estratificación social, con base en la obra de Blau y Duncan (Jorrat, 2000: 105), adoptó un modelo general, el cual está relacionado fundamentalmente con las siguientes variables: categoría ocupacional o clase, status o prestigio ocupacional, ingresos y años de escolaridad; esas variables, sin lugar a dudas, continúan siendo de gran relevancia para el abordaje de investigaciones vinculadas a esta temática. Crompton (1994) señala que hoy debe considerarse, además, la variable sexo debido a la incorporación creciente de las mujeres al mercado laboral.

En palabras de Cachón (1989), existe un solo paradigma para abordar el problema de la estratificación social: el paradigma liberal, el cual se fundamenta teóricamente en el funcionalismo, situación que es un tanto extrema al considerar las líneas de investigación desarrolladas actualmente desde la perspectiva neoweberiana por Goldthorpe (2000, 2002), Goldthorpe y Mc Kninght (2004), así como desde la concepción neomarxista en la cual destacamos a Wright (1993). 
Retomando nuestra revisión del enfoque funcionalista, se puede señalar que su prolongada presencia como perspectiva teórica para el abordaje de la temática de la estratificación social no está exenta de dificultades; de hecho, es posible apreciar 4 obstáculos en la adopción de este enfoque. El primero de ellos guarda relación con el concepto de realidad, pues esta es asumida como un hecho transparente. El segundo obstáculo centra la atención en la tendencia a la homogenización de las situaciones a partir de la consideración de la sociedad como un mercado único. Un tercer obstáculo está vinculado a la 'unidad de análisis', pues no resulta fácil discriminar entre la elección del individuo y/o la familia como unidad de análisis en los estudios de estratificación. Por último, el cuarto obstáculo alude a la concepción probabilista de la movilidad social, es decir, la igualdad de oportunidades como un principio naturalizado. De acuerdo con Fernández:

La discusión en torno a la estratificación se refiere al sistema de posición, no a los individuos que ocupan dichas posiciones. Una cosa es preguntarse por qué diferentes posiciones llevan consigo diferentes grados de prestigio, y otra completamente distinta es preguntar cómo ciertos individuos consiguen dichas posiciones (...) Ambas cuestiones están relacionadas, sin embargo es necesario mantenerlas separadas en nuestro pensamiento (Fernández, 2001: 132).

Por su parte, Davis y Moore (2001) sostienen que todo sistema de estratificación se genera a partir de principios fundamentales, tales como los siguientes: 1) Grado de especialización: este principio presupone que a medida que las sociedades crecen, se demanda la satisfacción de nuevas necesidades, que serán cubiertas solo en la medida en la que se prepare a los individuos para la ejecución de tareas cada vez más especializadas. Este proceso de sobreespecialización tendrá una repercusión directa, no en la estructura (mantiene el carácter piramidal), pero sí a nivel de los estratos que componen dicha estructura; 2) La naturaleza de la importancia funcional: en toda sociedad moderna se asume la necesidad de distribuir a los individuos dentro de la estructura social por cuanto se reconoce como la estrategia a utilizar para el mantenimiento y trascendencia de las sociedades; esta distribución, al interior de la estructura, supone ciertos niveles de especialización "productiva" que facilitará el proceso de distribución de la población al interior de esta; 3) Magnitud de las diferencias emulativas: referida a la distancia social entre posiciones, que teniendo en cuenta toda las escala debería conducir a una medida cuantitativa. En el marco de las distancias sociales operan una serie de variables como por ejemplo ingresos, escolaridad y lugar de residencia; 4) El grado de oportunidad: está vinculado con 
los aspectos dinámicos de la sociedad; precisamente, se refiere a la estructura de oportunidades que operan en una sociedad específica, esto implica que puedan clasificarse como sociedades abiertas, intermedias o cerradas. Esa clasificación depende de cuan posible es la movilidad de los individuos entre los estratos que componen la estructura social; 5) El grado de solidaridad de estrato: el grado de solidaridad de clase puede variar hasta cierto límite independientemente de los otros criterios, y por esto es un principio importante para clasificar sistemas de organización. Esto puede vincularse a los planteamientos de Parkin, ${ }^{1}$ quien afirma que la sociedad está configurada de acuerdo a grupos de status que se cierran ante el eventual riesgo de perder prestigio o posiciones de privilegio, y de Katzman ${ }^{2}$ cuando reconoce la variable residencial como elemento significativo para el enfrentamiento de situaciones de riesgo social, puesto que en el barrio se establecen las redes sociales necesarias para el fortalecimiento del capital social, 0 bien para el mantenimiento 0 debilitamiento del mismo.

El sistema de estratificación social de una población depende fundamentalmente de los elementos mencionados en los 5 criterios bases en los cuales se generan los diferentes modelos de sociedades. Todos los criterios entran en el juego de las relaciones para que en una sociedad se configure un sistema de estratificación social específico, lo que le otorga un carácter fuertemente interno; no obstante, también existen elementos externos (al propio sistema de estratificación) que pueden afectar el modelamiento de un sistema de estratificación para una sociedad puntual. Estos son: 1) El estado de desarrollo cultural: cuando la cultura crece, se hace necesario el aumento de la especialización, que contribuye, a su vez, al aumento de la movilidad, disminución de la solidaridad de estrato, y, a un cambio funcional; 2) Situación con respecto a otras sociedades: la estructura de clases de una sociedad se ve afectada por la presencia o ausencia de conflicto abierto con otras sociedades, relaciones de libre comercio, difusión cultural, etc.; 3) EI tamaño de la sociedad: una sociedad pequeña limita el grado al que puede llegar la especialización funcional, el grado de segregación de diferentes estratos y la magnitud de las desigualdades.

Tal como se mencionara, la obra de Blau y Duncan (1967) es la que permite (desde el funcionalismo clásico) forjar un modelo general de estratificación social. Los autores parten del supuesto de que el estudio de la estratificación social en la

\footnotetext{
Véase Feito (1997): "Estructura Social contemporánea: las clases sociales en los países industrializados".

2 Véase Katzman (1999): "Segregación Residencial y Desigualdades Sociales en Montevideo".
} 
sociedad moderna puede avanzarse mejor mediante la investigación sistemática del status ocupacional y la movilidad. El foco está en la jerarquía estratificada de ocupaciones más que en algún otro aspecto de la diferenciación social. Los autores asumen que ello no posibilita el análisis de las diversas dimensiones de la estratificación, sin embargo, afirman que la clase puede definirse en términos de recursos económicos e intereses, y el principal determinante de estos para la vasta mayoría de individuos es su posición ocupacional. Si bien se reconoce que no da cuenta de los distintos aspectos de la clase, es su mejor indicador único, al afirmar que hay una relación más estrecha entre clase económica y posición ocupacional que la que existe entre posición ocupacional y status de prestigio. El planteamiento central de los autores es concebir que la estructura ocupacional consiste en las relaciones entre sus subgrupos y que estos subgrupos ocupacionales (no los individuos que los componen) son las unidades de análisis. Con respecto a las clases ocupacionales, sostienen que son agrupamientos sociales significativos y no enteramente categorías arbitrarias. Sus miembros comparten oportunidades de vida y experiencias sociales y muchos contactos sociales directos de los individuos en el trabajo. De ahí la importancia de abordar el estudio de la estructura socio-ocupacional tomando como variables fundamentales la rama de actividad económica, los grandes grupos ocupacionales y sus respectivas categorías de ocupación.

Sobre la base de lo expuesto en las líneas precedentes, cabe señalar que el objetivo general de este trabajo fue determinar las transformaciones ocurridas en la estructura socio-ocupacional de la provincia de Ñuble en el período inter-censal 1992-2002, considerando como base las variables consignadas por la perspectiva funcionalista. Para ello, se abordaron los siguientes objetivos específicos: a) Identificar los cambios que experimenta la distribución de la Población Económicamente Activa (PEA) con respecto a la rama de actividad económica, la categoría y grupo de ocupación; b) Determinar la estructura ocupacional existente para cada año del período que comprende el estudio; y, c) Analizar los cambios ocurridos en la estructura socio-ocupacional en el período comprendido entre los años 1992 y 2002, desde la perspectiva funcionalista clásica. 


\section{Materiales y métodos}

La investigación fue de carácter cuantitativo de alcance descriptivo longitudinal. De acuerdo a los objetivos explicitados en el apartado anterior, se decidió plantear la siguiente hipótesis de investigación: "En el período inter-censal 1992-2002 la estructura socio-ocupacional de la provincia de Ñuble presenta una asalarización de su población económicamente activa, cuya importancia relativa se concentra en las ramas de actividad económica $\mathrm{F}$ (comercio por mayor y menor; y restaurantes y hoteles) y H (establecimientos financieros, seguros, bienes inmuebles y servicios prestados a la empresa)".

Henríquez (1990: 17) plantea que:

Las fuentes estadísticas, susceptibles de ser utilizadas para el estudio de las clases sociales, tienen algunas limitaciones y defectos que es preciso establecer, a fin de controlar los sesgos 0 errores a los que pueda dar lugar el uso acrítico de las mismas (...) La fuente documental básica para la determinación de las clases sociales y de las relaciones sociales de producción son los Censos de Población.

Si bien el autor se refiere al análisis de clases, su reflexión también es pertinente cuando se plantea la estratificación ocupacional, ya que, tal como se mencionara al inicio del presente trabajo, la estratificación social tiene como elemento fundamental de análisis el concepto de clases sociales, el cual puede ser entendido como "una denominación general para describir las estructuras de la desigualdad en las sociedades modernas" (Crompton, 1994: 41).

Concretamente, la investigación utilizó como fuente de información los Censos de Población y Vivienda de los años 1992 y 2002, debido a que los resultados del estudio del año 2012 no han sido validados pues se detectaron errores en su aplicación. Es importante mencionar que las variables estudiadas son las que se detallan a continuación: 


\begin{tabular}{|c|c|c|}
\hline $\begin{array}{l}\text { Las ramas de actividad } \\
\text { económica }\end{array}$ & $\begin{array}{l}\text { Las categorías } \\
\text { ocupacionales }\end{array}$ & $\begin{array}{l}\text { Los grandes grupos de } \\
\text { ocupación }\end{array}$ \\
\hline $\begin{array}{l}\text { - A (agricultura, caza, } \\
\text { silvicultura y pesca). } \\
\text { - B (explotación de minas } \\
\text { y canteras). } \\
\text { - C (industrias } \\
\text { manufactureras). } \\
\text { - D (electricidad, gas y } \\
\text { agua). } \\
\text { - E (construcción). } \\
\text { - F (comercio por mayor } \\
\text { y menor; restaurantes y } \\
\text { hoteles). } \\
\text { - G (transporte, } \\
\text { almacenamiento y } \\
\text { comunicaciones). } \\
\text { - H (establecimientos } \\
\text { financieros, seguros, } \\
\text { bienes inmuebles y } \\
\text { servicios prestados a la } \\
\text { empresa). } \\
\text { - I (servicios comunales, } \\
\text { sociales y personales). }\end{array}$ & $\begin{array}{l}\text { - Asalariado. } \\
\text { - Servicio doméstico. } \\
\text { - Trabajador por cuenta } \\
\text { propia. } \\
\text { - Empleador o patrón. } \\
\text { - Familiar no remunerado. }\end{array}$ & $\begin{array}{l}\text { - } 0 \text { (profesionales } \\
\text { técnicos y personas en } \\
\text { ocupaciones afines). } \\
\text { - } 1 \text { (gerentes, } \\
\text { administradores y } \\
\text { funcionarios de categoría } \\
\text { directiva). } \\
\text { - } 2 \text { (empleados de } \\
\text { oficina y personas en } \\
\text { ocupaciones afines) } \\
\text { (intelectuales, científicos, } \\
\text { docentes en educación } \\
\text { superior). } \\
\text { - } 3 \text { (vendedores } \\
\text { y personas en } \\
\text { ocupaciones afines). } \\
\text { - } 4 \text { (agricultores, } \\
\text { ganaderos, pescadores, } \\
\text { cazadores, madereros). } \\
\text { - } 5 \text { (conductores de } \\
\text { medios de transporte } \\
\text { y personas en } \\
\text { ocupaciones afines) } \\
\text { - } 6 \text { (artesanos y operarios } \\
\text { en ocupaciones } \\
\text { relacionadas con } \\
\text { hilandería, la confección } \\
\text { del vestuarios y calzado, } \\
\text { carpintería, la industria } \\
\text { de la construcción, la } \\
\text { mecánica y las artes } \\
\text { gráficas). } \\
\text { - X (obreros y jornaleros) }\end{array}$ \\
\hline
\end{tabular}

Fuente: Censos de Población y Vivienda. INE.

Diagrama de elaboración propia. 
El análisis llevado a cabo fue de carácter descriptivo. Se integraron las variables para dar cuenta de las transformaciones generales acaecidas en la provincia de Nuble en el período inter-censal 1992-2002. Concretamente, los cálculos realizados se trabajaron con base en la Tasa de Crecimiento Aritmética o de Crecimiento Lineal:

$$
r:(\mathrm{Nf}-\mathrm{Ni}) /((\mathrm{Nf}+\mathrm{Ni}) / 2))^{*} 1 / \mathrm{t}^{*} \mathrm{k}
$$

\section{Resultados $^{3}$}

A nivel regional, con respecto a la concentración de Población Económicamente Activa (PEA), se aprecia que la provincia de Concepción presenta un incremento de su importancia relativa, al concentrar en el año 2002 el 50\% de la PEA; por su parte las provincias de Bío-Bío y Arauco no tuvieron variación en el período inter-censal 1992-2002, manteniendo el 19\% y $8 \%$ respectivamente de la PEA, a diferencia de la provincia de Nuble, la cual pierde importancia relativa en el contexto regional, al pasar de un $24 \%$ (1992) a un $23 \%$ aproximadamente (2002).

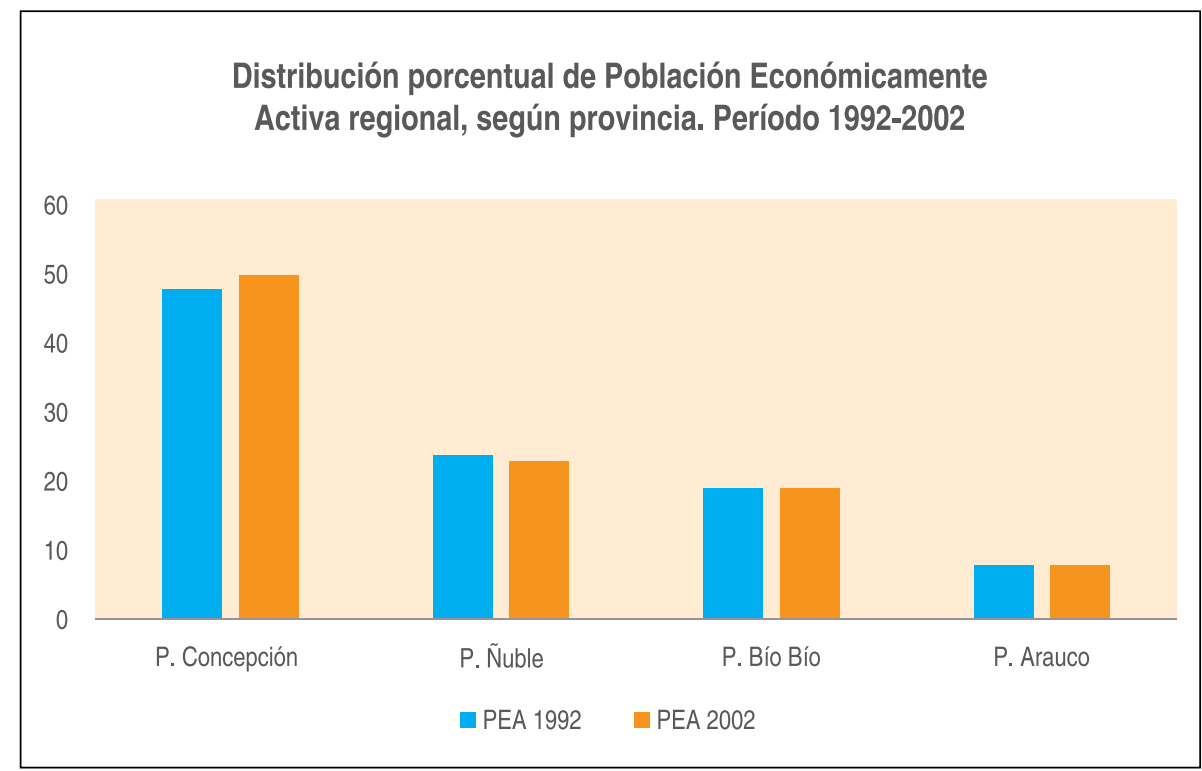

Gráfico 1

3 Todos los gráficos expuestos son de elaboración propia. 
A nivel de las Ramas de Actividad Económica (RAE) se evidencian cambios de importancia, en cuanto a la influencia relativa de cada una de ellas. En efecto, al apreciar la RAE A (correspondiente a agricultura, caza, silvicultura y pesca) es posible constatar que la PEA disminuye considerablemente, al pasar de 45.214 individuos en 1992, a 28.024 en el año 2002, lo que en términos generales significó una pérdida de importancia relativa de la rama en el contexto provincial, concretamente se visualiza un promedio anual de decrecimiento de $4.7 \%$. Esa situación es de gran relevancia, puesto que en el contexto provincial la actividad productiva fundamental, históricamente, estuvo asociada a rubros primarios extractivos, particularmente de la agricultura; situación que estaría en proceso de transformación, al reconocerse el incremento que tuvieron tres ramas específicas. En efecto, las ramas que presentan un incremento en su importancia relativa en la provincia y que consecuentemente expresan un importante crecimiento intercensal fueron: la RAE $H$ (establecimientos financieros, seguros, bienes inmuebles y servicios prestados a la empresa), RAE E (construcción) y RAE D (electricidad, gas y agua), que tuvieron un crecimiento promedio anual en el período de $8.8 \%$, $4.7 \%$ y $4.0 \%$ respectivamente (ver gráfico 2 ).

Sin lugar a dudas, llama la atención el importante incremento que posee la RAE $\mathrm{H}$, situación que expresaría el proceso de consolidación que han tenido las reformas neoliberales implementadas a mediados de los 70 , y que evidenciaría la adscripción y transición de la provincia de Ñuble hacía un tipo de sociedad postindustrial capitalista globalizada (Garretón, 2000), consecuentemente con lo que ocurre en el contexto nacional. Por su parte, el incremento reflejado en las RAE D y E puede explicarse, entre otras cosas, por las políticas de estabilización macroeconómica/macrosocial con la que comienzan a gobernar la Concertación de Partidos por la Democracia (Ffrench-Davis, 2001), por cuanto generan políticas orientadas a dar soluciones habitacionales y a ampliar servicios básicos tanto en el sector urbano como rural. 
Población Económicamente Activa, provincia de Ñuble, según Rama de Actividad Económica.

Años 1992-2002

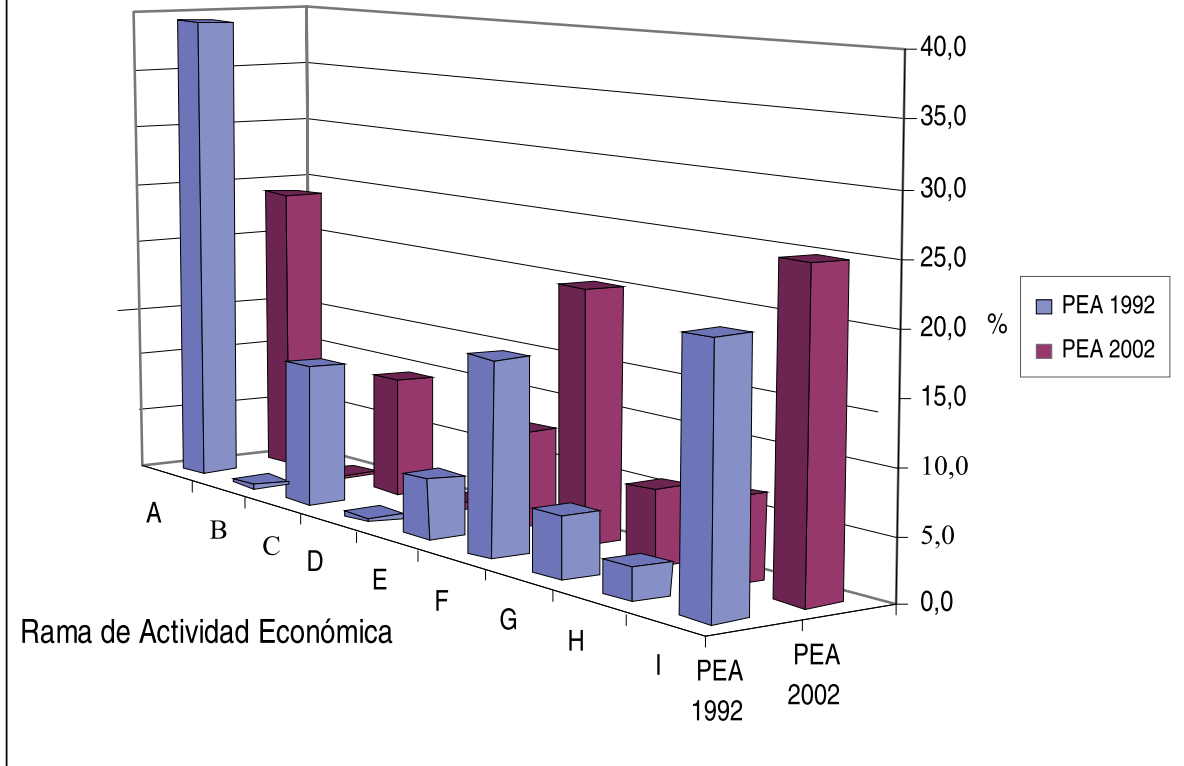

Gráfico 2

En lo que respecta al comportamiento de la PEA al interior de las Categorías Ocupacionales (CO), se constata que la categoría ocupacional que presenta el mayor incremento corresponde a los asalariados, con un crecimiento anual de un $1 \%$ aproximadamente, seguida de las personas dedicadas al servicio doméstico, cuyo incremento bordea el $0.3 \%$ en promedio anual; en el lado opuesto se aprecia que la categoría correspondiente a familiares no remunerados presenta un decrecimiento del orden de $5.7 \%$ en promedio anual, seguida de las categorías patrón empleador y trabajadores por cuenta propia con un promedio anual de $-2.4 \%$ y un $-0.4 \%$; respectivamente. En este escenario, considerando las categorías de los extremos, se puede afirmar que, a nivel provincial, los asalariados incrementan su importancia relativa, pasando de un $62 \%$ en 1992 a un $67 \%$ en el 2002; en cambio los familiares no remunerados pasan de concentrar un $7 \%$ de la PEA provincial en 1992, a un 4\% aproximadamente el 2002 (ver Gráfico 3). 


\section{Población Económicamente Activa, provincia}

de Ñuble,según Categorías Ocupacionales.

Años 1992-2002

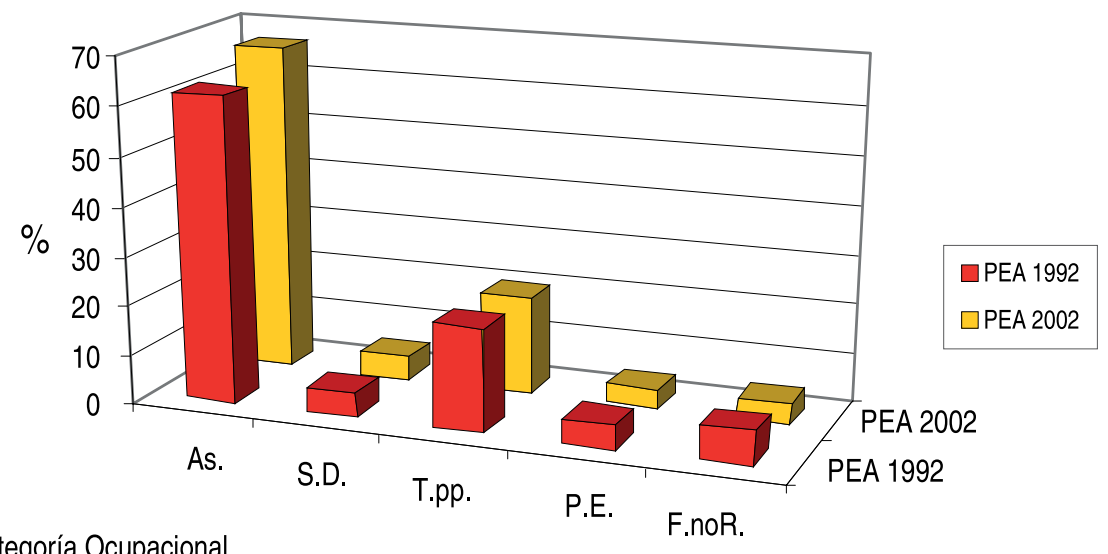

Categoría Ocupacional

Gráfico 3

Por su parte, en lo que respecta a los Grandes Grupos Ocupacionales (GO), a nivel provincial, se evidencia que si bien todos los GO manifiestan cambios, solo algunos de ellos presentan una variación significativa en términos de crecimiento inter-censal e importancia relativa en cuanto a concentración de PEA. Concretamente, el GO de mayor crecimiento ( $18.5 \%$ en promedio anual) corresponde al grupo $X$ (obreros y jornales), impactando directamente en su importancia relativa, la cual pasa de un $0.4 \%$ en 1992 , a un $9.7 \%$ aproximadamente en el 2002. El otro grupo que posee un incremento significativo es el GO 0 (profesionales técnicos y personas en ocupaciones afines), pues se evidencia un incremento anual en el período del orden del $6.6 \%$, cambiando consecuentemente su importancia relativa, así, en 1992 en este grupo se concentraba el $8.5 \%$ de la población económicamente activa provincial, y en el 2002, esta alcanza un 16.7\%. En el otro extremo, es decir, el GO que presenta un crecimiento negativo de importancia, corresponde al GO 4 (agricultores, ganaderos, pescadores, cazadores, madereros), con un promedio anual del orden de $-10.3 \%$, pasando de un 30.1\% de PEA en 1992, a un 9.5\% en el 2002 (ver Gráfico 4). 


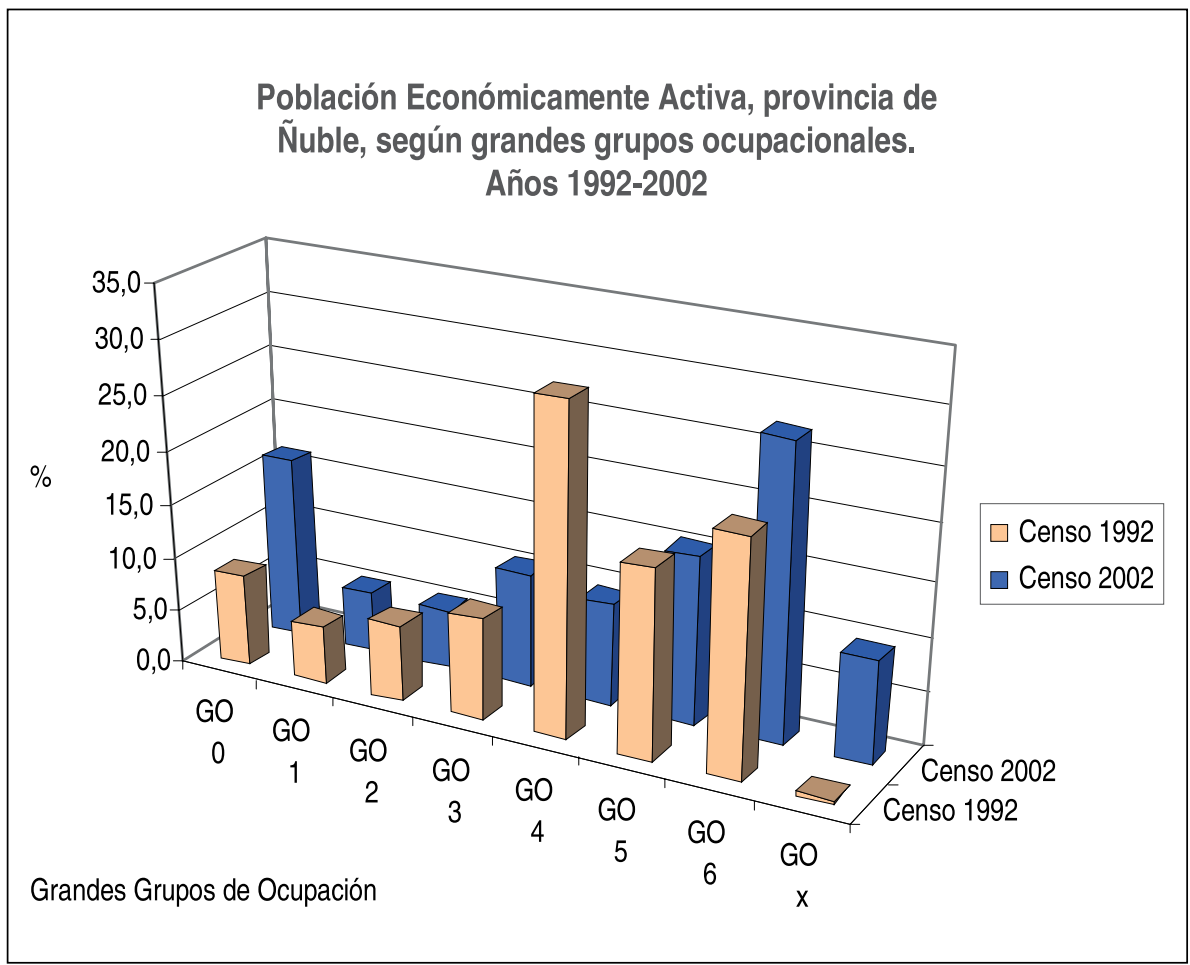

Gráfico 4

\section{CONCLUSIONES}

La pérdida de PEA en la RAE A (correspondiente a agricultura, caza, silvicultura y pesca) puede entenderse debido a la pérdida de población que presenta el área rural de la provincia, la cual, de acuerdo a datos proporcionados por el INE, pasa de 180.000 personas en 1992 a 152.995 en el 2002; situación que implica un crecimiento negativo del orden de un -1.62 en promedio anual durante el período inter-censal (1992-2002). En consecuencia, es posible reconocer una relación entre la pérdida de importancia relativa de dicha rama y los cambios acontecidos a nivel de los GO, concretamente el GO 4 (en el que se inscriben agricultores, ganaderos, pescadores, cazadores, madereros) y el GO X (al cual pertenecen obreros y jornales). En efecto el GO 4 es el que expresa la mayor pérdida de importancia relativa en cuanto a la concentración de PEA provincial, pasando de un $30.1 \%$ en 1992 a un $9.5 \%$ en el 2002; por su parte el GO X pasa de un $0.4 \%$ en 1992 a un $9.5 \%$. Ese comportamiento podría ser explicado, aunque no únicamente, por el proceso de migración ocupacional campo-ciudad. 
En lo que respecta a la hipótesis de investigación, puede señalarse que se apoya, por cuanto fue posible reconocer un proceso de asalarización. En efecto, se evidenció un incremento de la importancia relativa de esta categoría ocupacional en el contexto provincial, pasando de un $62 \%$ en 1992 a un $67 \%$ al finalizar el período de estudio; esto implicó un crecimiento promedio anual de la PEA en la CO asalariados, de un $1 \%$ aproximadamente. Concretamente, la RAE $\mathrm{H}$ (establecimientos financieros, seguros, bienes inmuebles y servicios prestados a la empresa) es la rama en la que se muestra el mayor incremento absoluto de la categoría, específicamente el crecimiento promedio anual en esta es de un 9.3\% aproximado, seguida de las ramas RAE E (construcción) con un 4.7\%, la RAE F (comercio por mayor y menor; y restaurantes y hoteles) con $3.6 \%$, la RAE G (transporte, almacenamiento y comunicaciones) con $2.2 \%$; y la RAE I (servicios comunales, sociales y personales) con aproximadamente un $2.1 \%$; el resto de las ramas presentan un decrecimiento en la $\mathrm{CO}$ asalariados.

Para finalizar, lo expuesto deja en evidencia que las variables ocupacionales utilizadas por la perspectiva funcionalista siguen representando una importante aproximación para la realización de investigaciones vinculadas a la temática de la estratificación social. En este sentido, es posible sostener que a nivel de la estructura social no se reconoce un cambio importante en la estratificación social de los sujetos en cuanto al tipo de labor desempeñada (manual o no manual), sino que fundamentalmente se constata un cambio en el rubro al que se encuentran asociados los individuos que se ubican en la base de la pirámide estratificacional, evidenciándose el segundo principio planteado por Davis y Moore (2001): el de la naturaleza de la importancia funcional. Ese postulado sostiene que en toda sociedad moderna se asume la necesidad de distribuir a los individuos dentro de la estructura social por cuanto se reconoce como la estrategia a utilizar para el mantenimiento y trascendencia de las sociedades; esta distribución al interior de la estructura supone ciertos niveles de especialización "productiva" que facilitarán el proceso de distribución de la población al interior de esta. Igualmente, a la luz de los datos censales, se constata que los asalariados corresponden a una categoría en extremo funcional para el mantenimiento de modelo de acumulación sustentado en el neoliberalismo, de esta forma, dados los cambios en la demanda de trabajadores lo que se reconoce es una movilidad ocupacional determinada por el rubro al que se encuentran asociados los individuos, mas no una movilidad ascendente en base a la categoría y el grupo ocupacional al que se adscriben los sujetos, pasando del rubro de carácter primario extractivo al de servicios de diferente índole. Además, se evidencia una transformación en cuanto al tipo de relación que establecen con las unidades productivas en las cuales se desempeñan. Así, a 
diferencia de lo que plantean autores como Portes y Hoffman (2003), Filgueira (2002), Klein y Tokman (2002), Martínez y León (2001) y Aquevedo (1998) en la provincia de Ñuble se reconoce una tendencia a la formalización en base a la asalarización de las labores desempeñadas por la PEA asociada al GO X (obreros y jornales).

\section{REFERENCIAS BIBLIOGRÁFICAS}

ATRIA, R. (2004). Estructura Ocupacional, estructura social y clases sociales. Serie Políticas Sociales, № 96 . Santiago: CEPAL.

BLAU, P. y DUNCAN, O. (1967). The American Occupational Structure. New York: John Wiley \& Sons, Inc.

CACHÓN, L. (1989). ¿Movilidad Social o Trayectorias de Clase? Madrid Siglo XXI.

CROMPTON, R. (1994). Clase y estratificación. Una introducción a los debates actuales. Madrid: Tecnos.

DAVIS, K; MOORE, W. (2001). Principios de estratificación social. En Fernández (Ed.) "Sociología de la Educación". Madrid: Ariel S.A.

FERNÁNDEZ, M., (2001). Sociología de la Educación. España: Ariel S.A.

FEITO, R. (1997). Estructura social contemporánea. Las clases sociales en los países industrializados. Madrid: Siglo XXI.

FILGUEIRA, C. (2001). La actualidad de viejas temáticas: sobre los estudios de clase, estratificación y movilidad social en América Latina. Serie Políticas Sociales 51. Santiago: CEPAL.

FRENCH-DAVIS, R. (2001). Entre el neoliberalismo y el crecimiento con equidad. Tres décadas de política económica en Chile. Santiago: Domen.

GARRETÓN, M. (2000). La sociedad en que vivi(re)mos. Santiago: Lom. 
GOLDTHORPE, J. (2000). Rent, Class Conflict and Class Structure: A Commentary on Sorensen. En The American Journal of Sociology; May; 105, 6. Proquest Social Science Journal, p. 1572.

GOLDTHORPE, J. (2002). Globalization and Social Class. West European Politics, Vol 25, № 3 (July 2002), pp 1-28.

GOLDTHORPE, J.; MC KNIGHT, A. (2004). The Economic Basis of Social Class. Centre of Analysis of Social Exclusion. London School of Economics. February.

HENRÍQUEZ, G. (1982). Aproximación Cuantitativa a la Estructura de Clases Regional. Informe de Investigación. Centro de Estudio para el Desarrollo Región del Bío-Bío. Chile.

HENRÍQUEZ, G. (1990). Metodología para el tratamiento de la información de fuentes estadísticas en el estudio de las clases sociales en el Ecuador. Revista IDIS, $N^{\circ}$ 26. Instituto de Investigaciones Sociales-IDIS. Universidad de Cuenca. Ecuador.

JORRAT, J. (2005) Aspectos descriptivos de la movilidad intergeneracional de clase en Argentina. Revista de Estudio sobre Cambio Social, año IV, N 17-18. Otoño/Invierno. https://www.aset.org.ar/congresos/7/04001.pdf

KATZMAN, R. (1999). Segregación Residencial y Desigualdades Sociales en Montevideo. Montevideo: CEPAL.

KLEIN, E.; TOKMAN, V. (2002). La estratificación social bajo tensión en la era de la globalización. Revista CEPAL № 72. Santiago.

MARTíNEZ, J. Y LEÓN, A. (2001). La estratificación social chilena hacia fines del siglo XX. Serie Políticas Sociales № 52. CEPAL, Santiago.

PORTES, A.; HOFFMAN, K. (2003). Las estructuras de clase en América Latina: Composición y Cambios durante la Época Neoliberal. Serie Políticas Sociales 68. CEPAL, Santiago.

WRIGHT, E. (1993). Reflexionando, una vez más, sobre el concepto de estructura de clases. En Carabañas, J y De Francisco, A (Comps.) "Teorías contemporáneas de las clases sociales". Pablo Iglesias, Madrid. 